EUROPHYSICS LETTERS

1 January 1996

Europhys. Lett., 23 (1), pp. 1-5 (1993)

\title{
Chaotic dynamics in shear-thickening surfactant solutions
}

\author{
RANJini Bandyopadhyay AND A. K. SoOd \\ Department of Physics, Indian Institute of Science, Bangalore 560 012, India
}

(received ; accepted )

PACS. .82.70.Gg- Gels and sols.

PACS. 83.50.Qm Thixotropy; thickening flows.

PACS. 83.50.By- Transient deformation and flow; time-dependent properties: start-up, stress relaxation, creep, recovery, etc.

\begin{abstract}
We report the observation of dynamical behaviour in dilute, aqueous solutions of a surfactant CTAT (cetyl trimethylammonium p-toluenesulphonate), below the overlap concentration $\mathrm{c}^{\star}$. At these concentrations, CTAT forms cylindrical micelles and shows a pronounced shear thickening transition above a concentration-dependent critical shear rate $\dot{\gamma}_{c}$. An analysis of the time-series of the stress relaxations at controlled shear rates in the shear-thickening regime shows the existence of correlation dimensions greater than two and positive Lyapunov exponents. This indicates the existence of deterministic chaos in the dynamics of stress relaxation at these concentrations and shear rates. The observed chaotic behaviour may be attributed to the stick-slip between the shear - induced structure (SIS) formed in the sheared surfactant solution and the coexisting dilute phase. At still higher shear rates, when the SIS spans the Couette, there is a transition to higher-dimensional dynamics arising out of the breakage and recombination of the SIS.
\end{abstract}

Introduction. - It is well known that surfactant molecules, under appropriate conditions, can assemble reversibly in solution to form various supramolecular structures. In very dilute solutions, at concentrations above the critical micelle concentration, these molecules selfassemble to form spherical micelles. Increasing the surfactant concentration results in the formation of cylindrical structures, which overlap and entangle to form clear viscoelastic solutions of giant wormlike micelles. At still higher concentrations, the surfactant solutions form mesophases exhibiting nematic, hexagonal or lamellar ordering. It is possible to pass from one phase to the other by simply changing the surfactant and cosurfactant concentrations, the salinity or the temperature of the system. The cylindrical structures that are formed at very low concentrations $c\left(c<c^{\star}, c^{\star}\right.$ is the overlap concentration), get entangled on shearing at a shear rate $\dot{\gamma}>\dot{\gamma}_{c}$ to exhibit a pronounced increase in viscosity [1, 2, 3]. At $\dot{\gamma}<\dot{\gamma}_{c}$, the surfactant solutions exhibit Newtonian flow behaviour. Recently, direct imaging studies of light scattered by the shear-thickening CTAB/ NaSal and TTAA/ NaSal have shown the existence of unstable shear induced structures/ phases (SIS/ SIP) [2, 3], which fracture at very high shear rates, giving rise to shear thinning [3]. Direct imaging of the SIS using freeze-fracture electron microscopy shows that these structures span hundreds of microns and have sponge-like textures [4]. 
In this paper, we present an experimental study on the nonlinear dynamics of stress relaxation in dilute solutions of the surfactant CTAT, at concentrations below the overlap concentration $\mathrm{c}^{\star}$. At these concentrations, CTAT forms cylindrical micelles [5] and undergoes a shear-thickening transition when sheared at $\dot{\gamma}>\dot{\gamma}_{c}$, which is followed by shear-thinning at higher values of $\dot{\gamma}$ [1]. The phase diagram and flow behaviour of aqueous solutions of CTAT in the linear and nonlinear regimes have been investigated extensively by Soltero et al. [5]. Previous investigations of the flow behaviour of shear-thickening solutions of CTAT [i] using rheometry and neutron scattering have shown the coexistence of a highly viscoelastic shear-induced phase (SIP) with a viscous regime made up of short aggregates. Oscillations in the apparent viscosity (and hence the stress) of dilute CTAT at controlled shear rates in the shear thickened regime have been observed by Gamez-Corrales et al. [1]. Observations of viscosity oscillations in $\mathrm{CTAB} / \mathrm{NaSal}$ [2] and TTAA/NaSal [3] in water under controlled stress conditions have been explained in terms of the growth and retraction of the SIS that form in the sheared solution.

In a recent paper [6], we have established the existence of deterministic chaos [7] in the stress relaxation of shear-thinning CTAT solutions at concentrations $c>c^{\star}$. The oscillations in the normal and viscoelastic stresses were explained in terms of the stick-slip between shear bands [8] that form in the plateau region of the flow curve. Here, we extend our experiments to more dilute solutions of CTAT ( $c=11 \mathrm{mM}$, zero-shear viscosity $\eta_{\circ} \sim 2 \mathrm{mPas}, c^{\star} \sim 15 \mathrm{mM}$ ) and show that the occurence of coexisting phases in a sheared surfactant solution is a prerequisite for the existence of dynamical behaviour in its stress relaxation. In the following sections, we will demonstrate the existence of deterministic chaos in the stress relaxation of shear-thickened CTAT, possibly due to the stick-slip between the SIS with the coexisting dilute phases. To our knowledge, this is the first observation of deterministic dynamics in the stress relaxation of shear-thickening surfactant systems. The observed chaotic behaviour is followed by an increase in complexity of the dynamics of stress relaxation at higher shear rates due to the fracture of the SIS, which is accompanied by the formation of large vortices in the sheared solution [3].

Sample Preparation and Experimental Apparatus. - CTAT, purchased from Sigma Chemicals, Bangalore, India was used to prepare the samples by dissolving requisite amounts of CTAT in doubly distilled and deionised water. The sample containers were sealed and kept at $25^{\circ} \mathrm{C}$ and shaken frequently to accelerate homogenisation. By measuring the viscosities of CTAT solutions prepared at different concentrations as a function of the shear rate $\dot{\gamma}$, and extrapolating the values to $\dot{\gamma}=0$, we have estimated the overlap concentration $c^{\star}$ of CTAT in water to be $\sim 15 \mathrm{mM}$. The rheological measurements were made using a Rheolyst AR1000N stress-controlled rheometer at $\mathrm{T}=25^{\circ} \mathrm{C}$, in a concentric cylinder geometry with an conical end (outer cylinder diameter $=30 \mathrm{~mm}$, inner cylinder diameter $=27.66 \mathrm{~mm}$, immersed height $=$ $25.8 \mathrm{~mm})$.

Experimental Results. -

Figure 1 shows the flow curve of $11 \mathrm{mM}(0.5 \mathrm{wt} . \%)$ CTAT at $\mathrm{T}=25^{\circ} \mathrm{C}$, measured by allowing 30 seconds between the acquisition of successive data points. The flow curve shows all the distinct regimes seen in TTAA/NaSal by $\mathrm{Hu}$ et al. in stress-controlled experiments [3], viz., a Newtonian region of constant viscosity (Regime I) followed by a shear thickening regime where the SIS nucleates heterogeneously from the inner wall of the Couette cell (Regime II). In regime II, the shear rate is seen to decrease with stress for a controlled stress measurement, which supports the idea of coexistense between the SIS and the dilute phase at these shear rates [1]. Next, we observe a shear-thickened regime where the stress increases almost linearly with shear rate (Regime III). In this regime, the SIS forms a percolating cluster that undergoes 
plug flow under shear and nucleates homogeneously in the sheared solution [3]. Regime IV is a shear thinning regime arising out of the fracture of the SIS at very high shear rates.

Figure 2 shows the shear stresses measured on application of step shear rates in the regimes II, III and IV. The shear stresses show an initial increase followed by a chaotic time-dependence about a mean value after a time $\tau_{p}$. We would like to point out at this stage that the flow curve shown in Fig. 1 is not the true steady state flow curve and the data in regimes II and III are in all probability, transient. We come to this conclusion as we find from Fig. 2 that steady state is not reached even after shearing for 1500 seconds. We find that $\tau_{p} \sim \dot{\gamma}^{-\alpha}$, where $\alpha=1.8$, in contrast to $\alpha=1$ for dilute TTAA/NaSal solutions [3]. The emphasis of this work is on the analysis of the acquired time-series using standard non-linear dynamical methods. We prove the existence of deterministic dynamics in the stress relaxation of sheared CTAT for shear rates lying in regimes II and III, where we have derived finite values of the correlation dimensions and positive values of the Lyapunov exponents. The dynamics in regime IV shows a transition to higher degrees of complexity due to the stronger spatial dependence of the flow fields and a consequent coupling of the different wavevector modes, arising out of the percolation and fracture of the SIS.

Data Analysis. - We have employed the standard tools of nonlinear dynamics calculate certain metric and dynamical invariants [9] characterising the time-series measured in our experiments and displayed in Fig. 2. This is done to distinguish whether our signals arise out of stochastic or deterministic processes. As opposed to stochastic noise, chaotic dynamics may be predicted over short time scales and are extremely sensitive to initial conditions. The first invariant we have calculated is the correlation dimension $\nu$ which gives us an idea of the geometry of the attractor in phase space on which the stress trajectories lie in the asymptotic limit. Next, we have calculated the largest Lyapunov exponent $\lambda$ which provides a measure of the divergence of neighbouring trajectories. Presence of chaotic dynamics in a system requires $\nu>2$ and $\lambda>0$. The analysis is done by reconstructing the phase space by embedding the experimental time-series of shear stress $\sigma$ in $m$ dimensions using time delay vectors $L$ [9], so that the invariants associated with the dynamics may be calculated unambiguously. Let $\sigma_{j}=\sigma(j \Delta t)$ denote the time-series shown in Fig. 2, consisting of stresses measured at regular time intervals $\Delta \mathrm{t}(=1 \mathrm{~s})$, with $j=1$ to $\mathrm{N}(\mathrm{N}=1500)$, at various shear rates maintained constant during the duration of the experiment. A reasonable value of $L\left(=L_{\circ}\right)$ has been estimated for each time-series by plotting the stress autocorrelation function $C(\tau)$ versus $\tau$. The value of $\tau$ at which $C(\tau)$ falls to $\frac{1}{e}$ of its maximum value of 1 (at $\tau=0$ ) is used as an estimate of the optimum delay time $L_{\circ}$. An optimal value of the minimal embedding dimension $m_{\circ}$ has been estimated using the method of false nearest neighbours proposed by Kennel et al. [10, 11]. The values of $L_{\circ}$ thus obtained are used to construct the sequence of vectors $\left\{\vec{X}_{i}: i=1,2, \ldots . \mathrm{N}\right.$ $\left.(m-1) L_{\circ}\right\}$ which describe trajectories in $m$-dimensional phase space arising out of the dynamics $F: \vec{X}_{i} \rightarrow \vec{X}_{i+1}$, and which are used for the computation of the invariants characterising the dynamics such as the correlation dimension $\nu$ and the Lyapunov exponent $\lambda$ [9]. The calculations of the correlation dimension $\nu$ uses the algorithm of Grassberger and Procaccia 112 which involves the calculation of the correlation integral $C(R)$. The correlation integral $\mathrm{C}(\mathrm{R})$ is defined in an $m$-dimensional phase space as $C(R)={ }_{N \rightarrow \infty} \frac{1}{N^{2}} \sum_{i, j=1}^{N} H\left(R-\left|\vec{X}_{i}-\vec{X}_{j}\right|\right)$, where $\mathrm{H}(\mathrm{x})$ is the Heaviside function and $\left|\vec{X}_{i}-\vec{X}_{j}\right|$ is the distance between the pair of points $(i, j)$ in the $m$-dimensional embedding space. The sum in the expression for $C(R)$ gives the number of point pairs separated by a distance less than $\mathrm{R}$. For small $R \mathrm{~s}, C(R)$ is known to scale as $C(R) \sim R^{\nu}$, where $\nu$ is the correlation dimension [12]. A plateau in the plot of $\nu$ versus $\log (R)$ gives the correct $\nu$ for a chosen embedding dimension $\mathrm{m}$. If the attractor is unfolded by choosing a large enough $m$, then the correlation dimension $\nu$ becomes independent of the value 
of the embedding dimension $m$. The value of $m=m_{\circ}$ for which this independence sets in is the correct embedding dimension, and the corresponding $\nu$ is the correlation dimension. If an experimental signal satisfies $\nu<m$, then the signal is due to deterministic chaos rather than random noise [12]. Figure 3 (a) shows a plot of $\nu$ versus $\log (R)$ for $m=1-6$ (increasing in the direction shown by the arrow) at $\dot{\gamma}=60 \mathrm{~s}^{-1}$, which corresponds to regime II of the flow curve. The value of $\nu$, obtained from the plateau in the $\nu$ versus $\log (R)$ plot, is found to saturate to a value of 2.6, which gives $m_{\circ} \sim 3$ or 4 . The inset shows the values of the correlation dimensions $\nu$ versus the embedding dimensions $m$ calculated from the experimental data (open circles, marked as E) and five sets of surrogate data (SI-SV) generated by randomising the phases of the original data [10]. The values of $\nu$ calculated from the surrogate data do not converge even at $m=6$ and lie well above the values calculated from the experimental time-series. This implies the presence of deterministic dynamics in the stress relaxation of shear-thickening CTAT at $\dot{\gamma}=60 \mathrm{~s}^{-1}$.

Figure 3(b) shows the plot of $\nu$ versus $\log (R)$ for the data set acquired at $\dot{\gamma}=400 \mathrm{~s}^{-1}$ which saturates to an approximate plateau value of 4.5 for $m \geq 6$ over a range of $\log (R)$. The values of $\nu$ calculated from the experimental and surrogate time-series are not very different in this case. These large values of $\nu$ and $m_{\circ}$ calculated for $\dot{\gamma}=400 \mathrm{~s}^{-1}$ may be interpreted as a manifestation of the increased complexity of the dynamics of the stress relaxation in regime IV arising out of the fracture of the SIS which is accompanied by the formation of vortices in the sheared solution. Figure 4 shows a plot of $\nu$ versus $\dot{\gamma}$ for the regimes II, III and IV. Figure 5 shows the calculation of the largest Lyapunov exponent for $\dot{\gamma}=40,50$ and $90 \mathrm{~s}^{-1}$, following the algorithm of Gao and Zheng [13]. This is done by defining $d_{i j}(k)=\left\|\vec{X}_{i+k}-\vec{X}_{j+k}\right\|$, the Euclidean distance between two vectors constructed using the embedding theorem by $k$ iterations of the dynamics $\mathrm{F}$, and plotting $\Lambda=<\ln \left[d_{i j}(k) / d_{i j}(0)\right]>$ as a function of $k$. The Lyapunov exponents, calculated by using the relation $\lambda=S / \Delta t \ln (2)$, where $\mathrm{S}$ is the slope of the plot, are found to be positive at all shear rates in regimes II and III, which confirms the existence of deterministic chaos at these shear rates. Due to the limited extent of our time-series, we have been unable to obtain reliable values of $\lambda$ for shear rates lying in regime IV. Preliminary analysis indicates an increase in the values of $\lambda$ in regime IV from those obtained in regime III, confirming the existence of higher dimensional chaos in this regime. The inset of Fig. 5 shows the values of $\lambda$ at different shear rates in regimes II and III.

Conclusions. - In summary, we have proved the existence of deterministic chaos in the stress relaxation of dilute CTAT solutions in the shear-thickening regimes (Regimes II and III). The chaotic dynamics can arise out of the stick-slip between the SIS and the coexisting dilute phase in the sheared solution. In regime IV, where the spatial dependence of the flow fields becomes significant due to the percolation and fracture of the SIS, we have demonstrated an increase in the complexity of the dynamics, which is reflected by an increase in the optimal embedding dimension $m_{\circ}$, the correlation dimension $\nu$ and the Lyapunov exponent $\lambda$. Stress relaxation in dilute CTAT, therefore, provides yet another example of deterministic chaotic dynamics in a driven system arising out of the coupling between the lateral and normal degrees of freedom [6, 14]. We hope that our work will stimulate theoretical understanding of the nonlinear flow of dilute surfactant solutions in terms of nonlinear coupled equations having additional predictive capabilities.

Acknowledgements. - We thank Sriram Ramaswamy, P. R. Nott and V. Kumaran for the use of the rheometer.

\section{REFERENCES}


[1] R. Gamez-Corrales et al., Langmuir, 15 (1999) 6755; J. F. Berret et al., Europhys. Lett., 41 (1998) 677.

[2] C. Liu and D. J. Pine, Phys. Rev. Lett. , 77 (1996) 2121.

[3] Y. T. Hu, P. Boltenhagen and D. J. Pine, J. Rheol., 42 (1998) 1185; P. Boltenhagen et al., Phys. Rev. Lett., 79 (1997) 2359; P. Boltenhagen et al., Europhys. Lett., 38 (1997) 389.

[4] S. L. Keller, P. Boltenhagen, D. J. Pine and J. A. Zasadzinski, Phys. Rev. Lett. , 80 (1998) 2725.

[5] J. F. A. Soltero et al., Langmuir, 11 (1995) 3337; J. F. A. Soltero, E. Puig And O. Manero, Langmuir, 12 (1996) 2654; J. F. A. SOltero et al., Langmuir, 15 (1999) 1604.

[6] R. Bandyopadhyay, G. Basappa And A. K. Sood, Phys. Rev. Lett., 84 (2000) 2022.

[7] E. Отт, in Chaos in Dynamical Systems, (Cambridge University Press) 1993.

[8] N. A. Spenley, M. E. Cates and T. C. B. McLeish, Phys. Rev. Lett., 71 (1993) 939; P. D. Olmsted and C.-Y. D. Lu, Phys. Rev. E, 56 (1997) R55.

[9] H. D. I. Abarbanel et al., Rev. Mod. Phys., 65 (1993) 1331.

[10] R. Hegger, H. Kantz and T. Schreiber, Chaos, 9 (1999) 413.

[11] The TISEAN package is written by R. Hegger. H. Kantz and T. Schreiber and is publicly available at http://www.mpipks-dreseden.mpg.de// tisean.

[12] P.Grassberger and I. Procaccia, Phys. Rev. Lett., 50 (1983) 346.

[13] J. Gao and Z. Zheng, Phys. Rev. E , 49 (1994) 3807.

[14] M. G. Rozman, M. Urbakh and J. Klafter, Phys. Rev. Lett. , 77 (1996) 683. 
EUROPHYSICS LETTERS

\section{FIGURE CAPTIONS}

Fig. 1. - (a) shows a plot of the shear stress $\sigma$ while (b) shows a plot of the viscosity $\eta$ versus shear rate $\dot{\gamma}$. Depending on the behaviour of $\eta$ at different shear rates, the flow curve may be divided into four regimes : regime I where the surfactant solution shows Newtonian flow behaviour, regime II characterised by shear-thickening as a result of the nucleation of SIS, regime III where the SIS spans the Couette and regime IV which shows shear-thinning as a result of the fracture of the SIS. The filled circles represent the shear-rate controlled measurements while the open squares denote stress control.

Fig. 2. - Plots of the relaxation of the shear stress $\sigma$ at shear rates $\dot{\gamma}=(\mathrm{a}) 40$, (b) 50, (c) $60 \mathrm{~s}^{-1}$ (regime II), (d) 90, (e) $200 \mathrm{~s}^{-1}$ (regime III) and (f) $400 \mathrm{~s}^{-1}$ (regime IV).

Fig. 3. - The calculations of the correlation dimensions from the time-series of stresses at $\dot{\gamma}=$ (a) $60^{-1}$ and (b) $400 \mathrm{~s}^{-1}$. The insets show the calculations of $\nu$ for the experimental data set (denoted by E) and five sets of surrogate data ( SI-SV) generated according to the algorithm of Theiler et al. [11]. $\nu$ saturates to 2.6 for the experimental data at $\dot{\gamma}=60^{-1}$. At $\dot{\gamma}=400^{-1}$, the $\nu$ 's calculated from the experimental data and the surrogate data sets are not very different, which indicates a transition to higher-dimensional dynamics.

Fig. 4. - Plot of $\nu$ versus $\dot{\gamma}$, which shows a monotonic increase of $\nu$ versus $\dot{\gamma}$.

Fig. 5. - Calculations of $\Lambda$ versus $k$ for $\dot{\gamma}=40,50$ and $90 \mathrm{~s}^{-1}$. The Lyapunov exponents $\lambda$ which may be estimated from the slopes of the plots, are found to increase with $\dot{\gamma}$, as shown in the inset. 


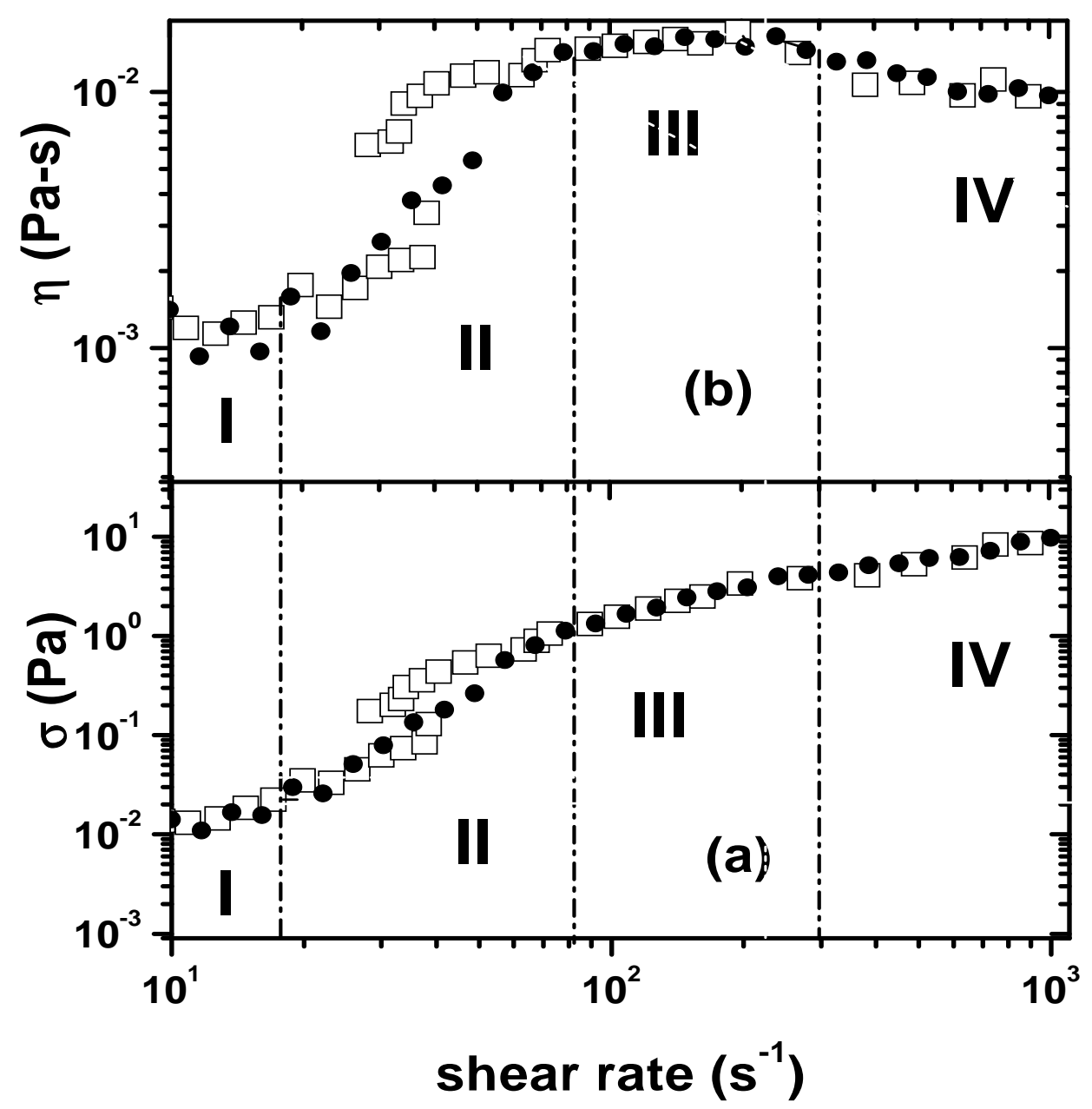

Figure 1 


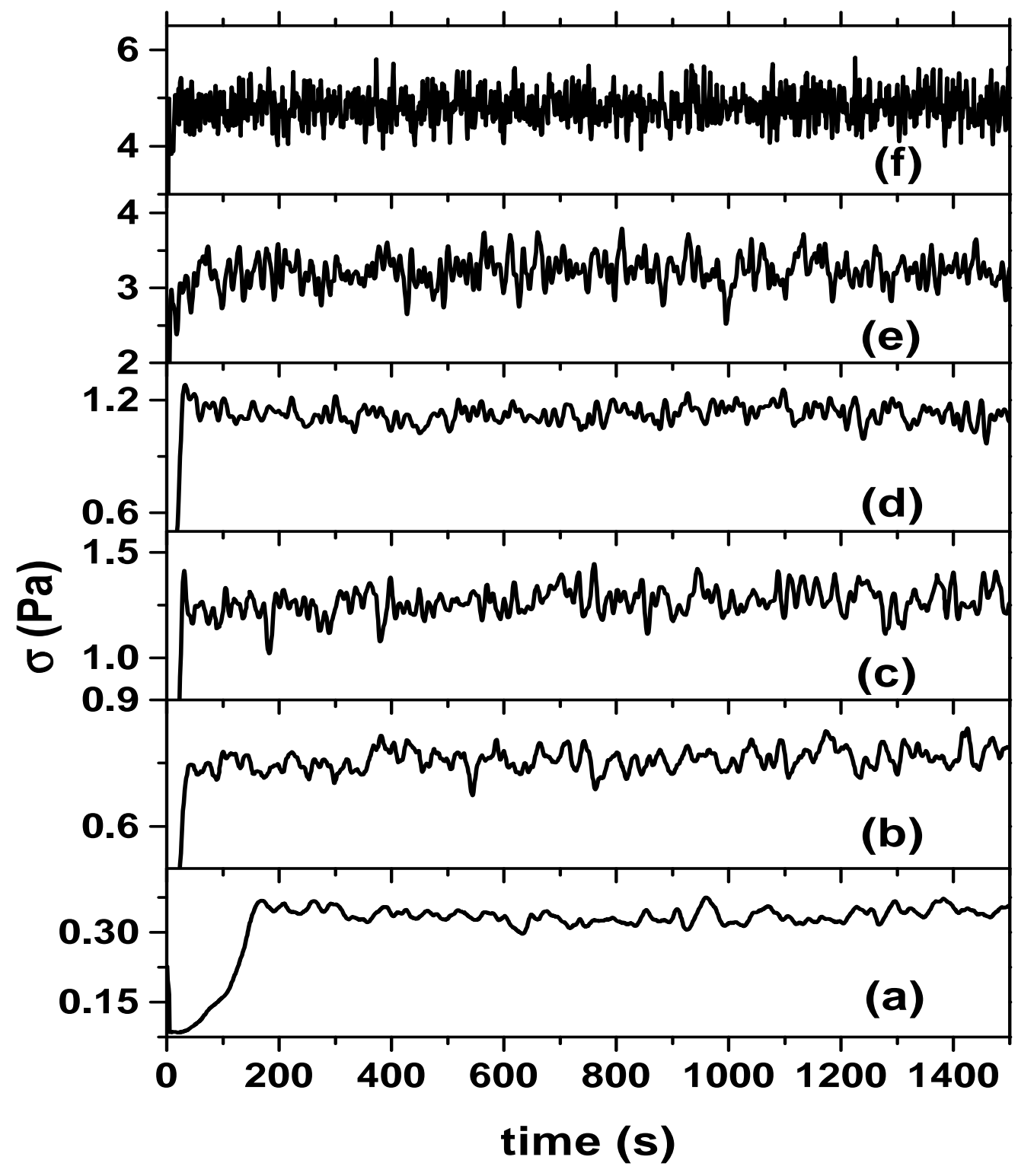

Figure 2 


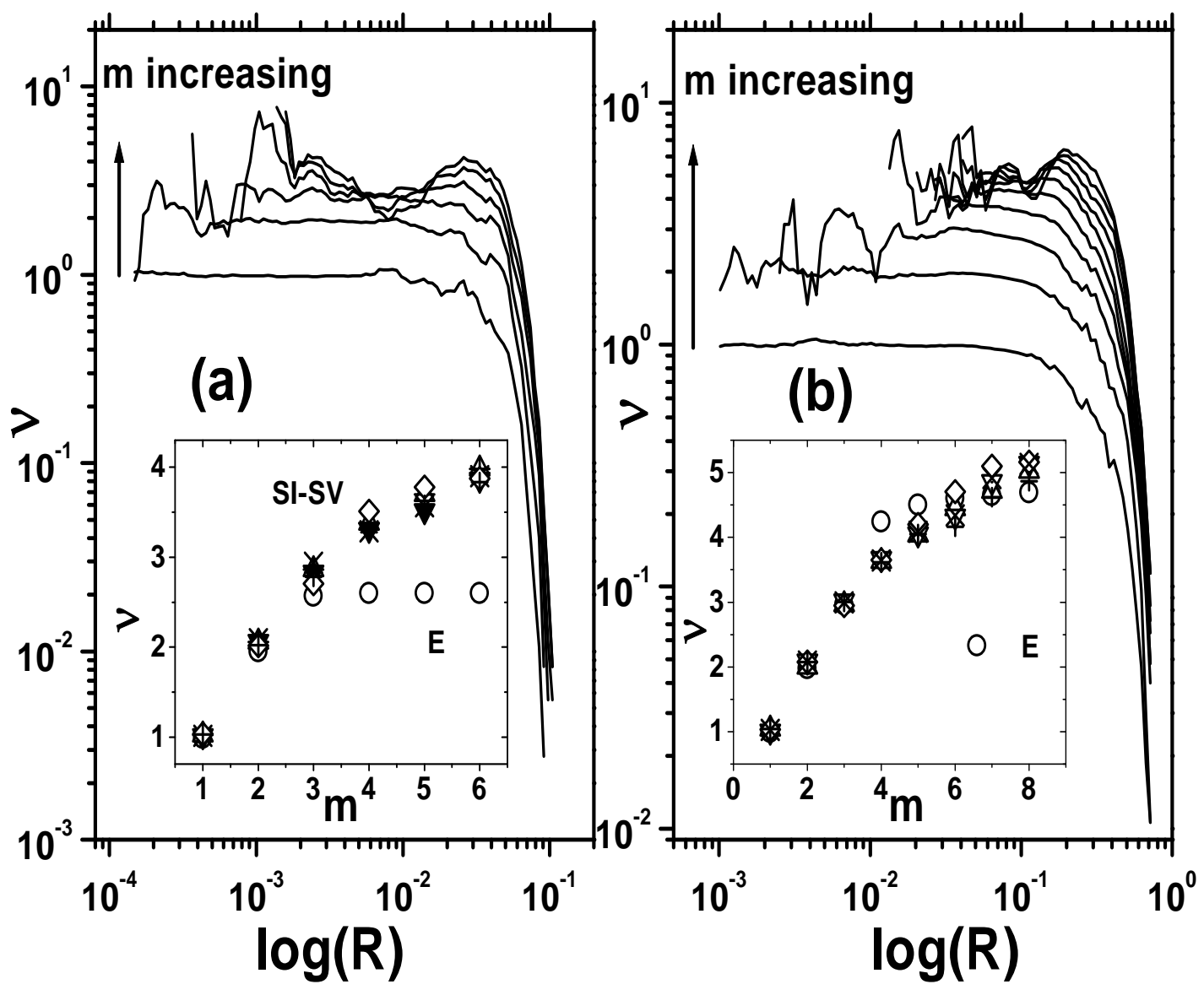

Figure 3 


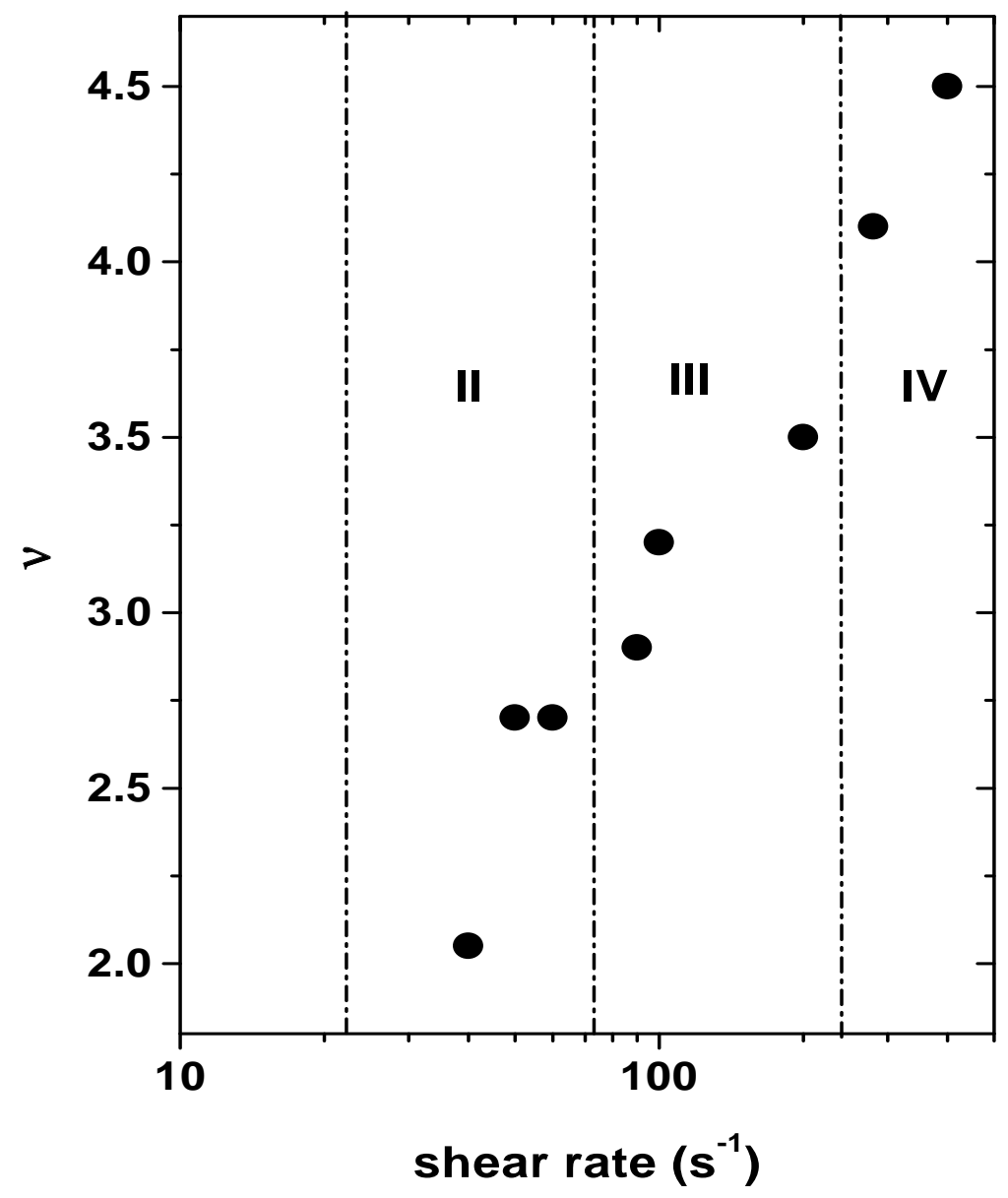

Figure 4 


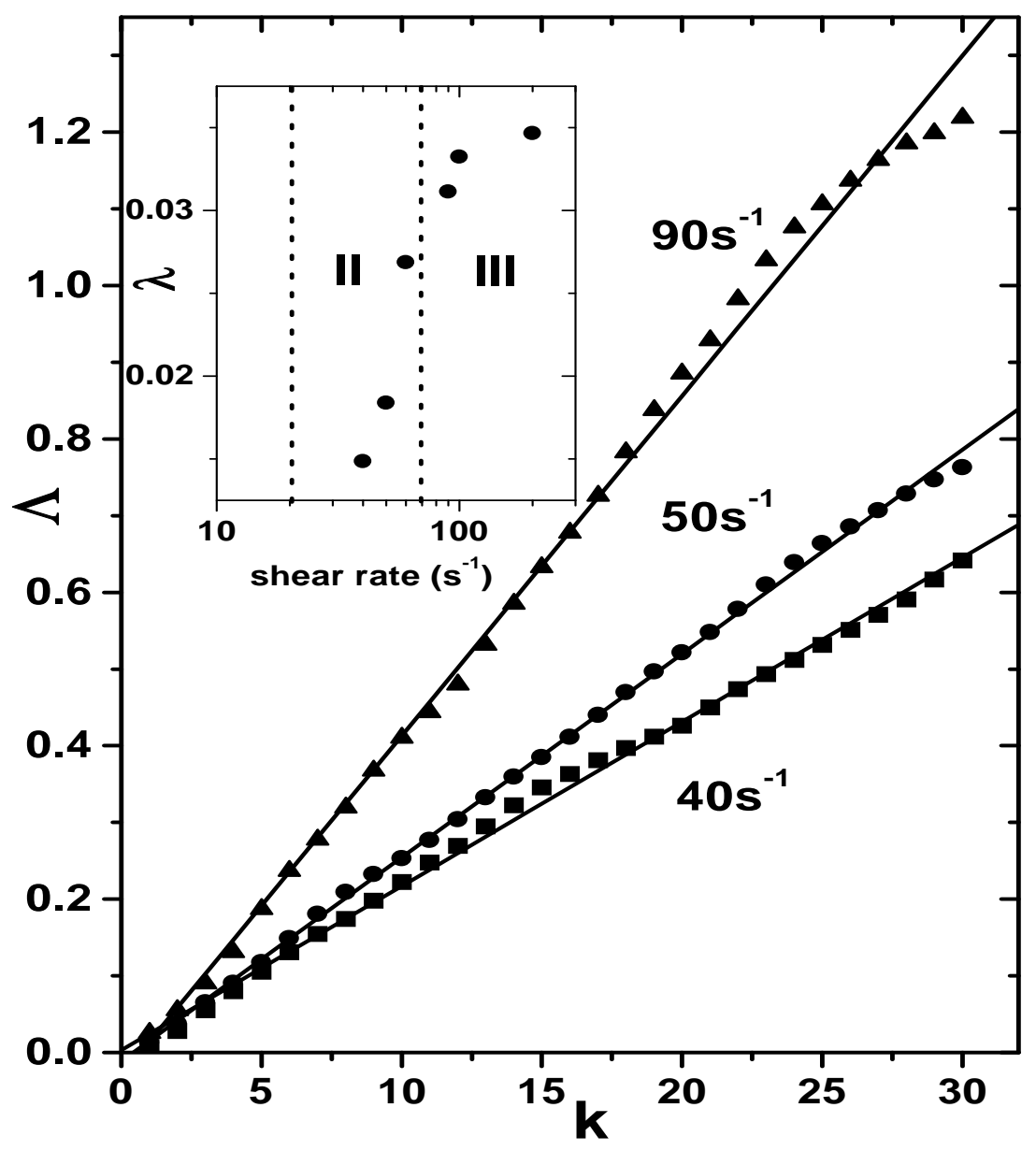

Figure 5 